\title{
Avaliação de Bases de Medidas considerando sua Aplicabilidade ao Controle Estatístico de Processos de Software
}

\author{
Monalessa Perini Barcellos ${ }^{1,2}$, Ana Regina Cavalcanti da Rocha ${ }^{1}$ \\ ${ }^{1}$ COPPE/UFRJ - Universidade Federal do Rio de Janeiro \\ Caixa Postal: 68511 - CEP: 21945-970 - Rio de Janeiro, RJ, Brasil \\ ${ }^{2}$ UFES - Universidade Federal do Espírito Santo - Centro Tecnológico \\ Departamento de Informática, CEP: 29075-910 - Vitória, ES, Brasil \\ monalessadinf.ufes.br; darocha@entroin.com.br
}

\begin{abstract}
Resumo. As exigências cada vez maiores do mercado de software levam as empresas a necessitarem de processos de software maduros, capazes de atender às demandas de qualidade e produtividade. A aplicação do controle estatístico na análise de desempenho de processos utiliza dados coletados ao longo dos projetos para analisar o comportamento dos processos da organização, identificando as ações necessárias para a estabilização e melhoria desses processos. Um elemento essencial para a aplicação do controle estatístico de processos é a adequação das medidas utilizadas. Este artigo apresenta um instrumento para avaliação de bases de medidas considerando sua aplicabilidade ao controle estatístico de processos.
\end{abstract}

Abstract. The escalating demands on the development of software products require software organizations to produce mature software processes that are capable of providing the required levels of quality and productivity. The use of statistical process control in performance process analysis use data collected during the course of the project to analyze the behavior of organization processes, identifying actions that are needed for the stabilization and improvement of those processes. An essential element for the statistical process control application is the suitability of the metrics being used. This article presents an instrument to the evaluation of statistical metrics schemes, considering their applicability in the statistical process control.

\section{Introdução}

As organizações buscam melhorar suas práticas de desenvolvimento e gerenciamento de projetos de software, a fim de aumentar sua competitividade no mercado. Nesse contexto, a gerência dos processos de software tem sido fator amplamente reconhecido para a melhoria dos processos e produtos das organizações de software [Fuggetta 2000].

Considerando a avaliação e melhoria de processos, exemplos notáveis de modelos e normas de apoio são o MPS.BR - Melhoria de Processo de Software Brasileiro (2007), o CMMI - Capability Maturity Model Integration (2006) e a norma internacional ISO/IEC 15504 - Avaliação de Processos de Software (2003). Em todas essas iniciativas, a medição ocupa papel fundamental no alcance e institucionalização dos programas de melhoria de processos de software.

A medição de software é considerada essencial no apoio à gerência e melhoria de processos e produtos de software [ISO/IEC FDIS 15939-E 2002]. Porém, a gerência 
e melhoria de processos baseada na medição "tradicional", que consiste na análise de medidas e a comparação destas, em um determinado ponto dos projetos, com os valores que foram planejados para aquele momento, não é suficiente para as organizações que buscam alta maturidade em seus processos. Nessas organizações, faz-se necessário conhecer o comportamento dos processos para determinar o seu desempenho em execuções anteriores e, a partir daí, prever o desempenho nos projetos correntes e futuros para analisar se serão capazes de atender os objetivos estabelecidos [Fenton et al. 2004].

A análise de desempenho de processos é realizada a partir da aplicação de métodos estatísticos aos dados coletados nos projetos, que alimentam o repositório organizacional de medidas para que seja realizada sua análise, sendo possível, a partir daí, identificar tendências e ações, não só no nível dos projetos, mas também no nível organizacional. A identificação de tendências e outros aspectos relativos ao desempenho e capacidade dos processos tornam-se a base concreta para a tomada de decisões que resultarão na melhoria dos processos.

A utilização do controle estatístico na análise de desempenho de processos requer que as organizações tenham alcançado um considerável nível de maturidade em seus processos de software, devendo possuir um conjunto de processos padrão definido e implementado e utilizar práticas de Engenharia de Software em seus projetos, incluindo a definição e coleta de medidas ao longo dos projetos e armazenamento destas em uma base organizacional de medidas [Sargut and Demirors 2006].

A análise de desempenho dos processos somente pode ser realizada se a organização possuir uma base com medidas válidas. Wang and Li (2005) argumentam que é muito difícil para as organizações definirem e coletarem medidas adequadamente, o que faz que as práticas necessárias às organizações de alta maturidade não sejam possíveis para a maioria das organizações de software.

Para que medidas adequadas à realização da análise de desempenho dos processos sejam definidas e coletadas, um programa de medição bem planejado e cuidadosamente focado deve ser definido [Kilpi 2001]. Porém, essa ainda é uma questão que requer atenção, pois vários estudos têm mostrado que as organizações, comumente, definem programas de medição precários, que não produzem medidas que permitam a análise do desempenho e capacidade de seus processos [Wang and Li 2005] [Kitchenham et al. 2006] [Sargut and Demirors 2006] [Komuro 2006].

No contexto dos problemas relacionados às medidas, que dificultam a realização do controle estatístico de processos, este artigo apresenta um instrumento para avaliar bases de medidas considerando sua aplicabilidade ao controle estatístico dos processos. O artigo encontra-se assim organizado: na seção 2 é realizada uma breve fundamentação teórica sobre o controle estatístico de processos, destacando-se questões relacionadas à medição nesse contexto; na seção 3 é apresentado o instrumento de avaliação definido; na seção 4 são apresentados os resultados de aplicação do instrumento às bases de medidas de duas organizações; e, na seção 5, são realizadas as conclusões do artigo.

\section{Controle Estatístico de Processos}

O controle estatístico de processos foi originalmente desenvolvido para implementar um processo de melhoria contínua em linhas de produção na área de manufatura, envolvendo o uso de técnicas estatísticas e de resolução de problemas com o objetivo de detectar padrões de variação no processo de produção para garantir que os padrões de 
qualidade estabelecidos para os produtos fossem alcançados. É uma metodologia utilizada para determinar se um processo está sob controle, sob o ponto de vista estatístico [Lantzy 1992].

Apesar de sua utilização na análise de desempenho e melhoria de processos não ser novidade para a indústria em geral, no contexto das organizações de software, pode ser considerado algo relativamente recente. Consequentemente existem, ainda, muitas dúvidas sobre sua aplicação [Card, 2004] [Komuro 2006] [Garcia et al. 2007].

Mesmo sendo considerada recente, é possível encontrar relatos de experiência e estudos no contexto da aplicação do controle estatístico a processos de software [Kilpi 2001] [Garcia et al. 2004] [Komuro 2006] [Sargut and Demirors 2006] [Tarhan and Demirors 2006] [Wang et al. 2006].

Analisando-se os relatos e estudos registrados na literatura, é possível perceber que há um gap entre os cenários organizacionais reais e os cenários desejáveis, propícios à implantação e realização efetiva do controle estatístico de processos. As experiências com a realização do controle estatístico de processos nas organizações têm revelado aos pesquisadores e profissionais de empresas um cenário caracterizado por problemas e situações não favoráveis ou impossibilitadoras da implantação/execução bem sucedida do controle estatístico de processos. Nesse aspecto, são apontadas diversas dificuldades, destacando-se: $(i)$ a não adequação das medidas coletadas pelas organizações à aplicação no controle estatístico de processos para que seja possível analisar o desempenho dos processos [Wheeler and Poling 1998] [Sargut e Demirors 2006] [Tarhan and Demirors 2006] [Boria, 2007] [Kitchenham et al. 2007]; (ii) a utilização inadequada dos métodos estatísticos necessários à estabilização e análise de desempenho de processos [Eickelman and Anant 2003] [Kitchenham et al. 2007]; e, (iii) as particularidades inerentes aos processos de software, o que exige uma adaptação dos conceitos do controle estatístico de processos tradicional (manufatura) à área de software [Lantzy 1992] [Sargut and Demirors 2006] [Komuro 2006] [Wang et al. 2006].

Considerando os problemas citados e focando as questões relacionadas à não adequação das medidas, Sargut and Demirors (2006) afirmam que há mais trabalho para preparar a estrutura necessária para realizar o controle estatístico de processos do que para realizar o controle estatístico propriamente dito, já que, na maioria das vezes, as organizações não alimentam suas bases de medidas com medidas válidas e aplicáveis ao controle estatístico de processos, o que retarda sua realização, uma vez que, primeiramente, deve ser realizada a adequação das medidas para, só então, aplicar as técnicas do controle estatístico de processos.

Uma organização pode minimizar o esforço e tempo despendidos na preparação para o controle estatístico de processos utilizando, desde o início, um programa de medição bem definido que oriente a alimentação da base de medidas organizacional com medidas aplicáveis ao controle estatístico de processos. Porém, considerando os estudos e experiências registrados, esse não tem sido um fato comum.

Boria (2007), analisando organizações que buscam alcançar os níveis de maturidade mais elevados do CMMI (2006), onde, a partir do nível 4, faz-se necessário o controle estatístico de processos para a análise de desempenho, afirma que aquelas que, durante a realização das atividades requeridas ao nível 3, simplesmente se preocupam em atender os requisitos das áreas de processo, acabam criando uma base de medidas inúteis ao pensamento estatístico exigido no nível 4. Segundo ele, dois dos principais obstáculos para a realização do controle estatístico de processos são: o 
acúmulo de dados incorretos, capturados em medidas inúteis definidas sem visar utilização futura, e a escassez de dados.

Wang et al. (2006) também consideram o problema da escassez de dados e Komuro (2006) destaca que mesmo quando há volume de dados, muitas vezes eles são inúteis, resultantes de medições em organizações que dão maior ênfase ao volume de dados coletados do que à qualidade desses dados.

Wheeler and Poling (1998) enfatizam a importância da qualidade dos dados armazenados na base de medidas da organização, pois, no controle estatístico de processos, todas as ações de melhoria são baseadas nas análises dos gráficos de comportamento dos processos, gerados a partir dos dados armazenados na base de medidas. Segundo eles, para aplicar o controle estatístico de processos é preciso construir uma 'fundação', ou seja, os processos devem ser caracterizados por medidas válidas e dados de qualidade que serão utilizados para analisar a previsibilidade dos processos.

\section{Avaliação de Bases de Medidas considerando sua Aplicabilidade ao Controle Estatístico de Processos}

Conforme apresentado na seção anterior, a literatura aponta que um dos maiores problemas para realizar o controle estatístico de processos de software se deve às dificuldades relacionadas às medidas coletadas e à base de medidas das organizações [Kitchenham et al. 2006] [Komuro 2006] [Boria 2007].

Considerando esse contexto, foi realizado um estudo baseado em revisão sistemática da literatura ${ }^{1}$ com o objetivo de identificar os principais fatores relacionados ao processo de medição e às medidas que influenciam, positiva ou negativamente, na implementação do controle estatístico de processos e, a partir desses fatores, identificar um conjunto de requisitos necessários às medidas/base de medidas considerando sua utilização no controle estatístico de processos ${ }^{2}$. Baseando-se nos resultados do estudo, um instrumento para avaliação de bases de medidas considerando sua aplicabilidade no controle estatístico de processos foi definido. O estudo realizado e o instrumento definido são apresentados a seguir.

\subsection{Estudo Baseado em Revisão Sistemática da Literatura}

A aplicação da revisão sistemática da literatura estabelece um processo formal e controlado para conduzir revisões de literatura, evitando a introdução de tendências que podem desvirtuar os resultados da pesquisa. A aplicação da revisão sistemática da literatura requer que seja seguido um conjunto bem definido e seqüencial de passos metodológicos segundo um protocolo de pesquisa desenvolvido apropriadamente [Biolchini et al. 2005]. Para a realização do estudo deste trabalho foi utilizado o processo de apoio à condução de estudos baseados em revisão sistemática definido em [Montoni 2007], que apesar de utilizar os conceitos básicos da revisão sistemática da literatura, não utiliza todo o rigor original proposto, uma vez que o objetivo não é revelar hipóteses, e sim, garantir uma boa cobertura para a pesquisa.

O processo utilizado é composto por três atividades:

\footnotetext{
${ }^{1} \mathrm{O}$ estudo foi realizado durante os meses de janeiro e fevereiro de 2008.

2 No estudo realizado, os fatores que contribuem negativamente para a implementação do controle estatístico de processos foram denominados "problemas" e os fatores que contribuem positivamente foram denominados "características".
} 
(i) Desenvolver o Protocolo: nesta atividade o pesquisador realiza a prospecção sobre o tema de interesse do estudo definindo o contexto no qual o estudo será realizado e o objeto de análise. Em seguida, o protocolo que será utilizado como guia na execução do estudo deve ser definido, testado para verificar sua viabilidade e avaliado, identificando-se as alterações necessárias (se pertinente).

(ii) Conduzir a Pesquisa: nesta atividade o pesquisador executa a pesquisa coletando e armazenando os dados segundo o protocolo definido. Em seguida, análises quantitativas e qualitativas devem ser realizadas com base nos dados coletados.

(iii) Relatar Resultados: nesta atividade o pesquisador empacota os resultados gerados ao longo da execução do estudo, devendo estes ser publicados em alguma conferência, revista ou biblioteca de trabalhos científicos.

Na atividade Desenvolver o Protocolo, o protocolo de pesquisa foi definido e testado, tendo sido verificada sua viabilidade para a execução integral da pesquisa. Para a realização da pesquisa, foram consideradas publicações de estudos relacionados ao controle estatístico de processos que relatem problemas e/ou características relacionados à medição e/ou medidas que influenciam na realização do controle estatístico de processos, bem como publicações que descrevam estudos ou experiências de aplicação do controle estatístico de processos (nessas, os problemas e/ou características foram identificados durante a análise do conteúdo da publicação).

A não limitação a publicações que relatem, explicitamente, os problemas e/ou características relacionados à medição e/ou medidas que influenciam na realização do controle estatístico de processos se deu, pois, apesar do crescente número de publicações relacionadas à aplicação do controle estatístico a processos de software, o foco dessas publicações ainda é limitado a evidenciar a possibilidade e vantagens dessa aplicação ou a propor abordagens utilizadas em processos de software baseadas nos princípios do controle estatístico. Sendo assim, considerar apenas as publicações que relatem explicitamente os problemas e/ou características relacionadas à medição e/ou medidas que influenciam na realização do controle estatístico de processos poderia significar uma redução considerável de publicações analisadas.

$\mathrm{Na}$ execução da pesquisa foram consideradas como fontes as seguintes bibliotecas digitais: Scopus, Compendex, IEEE e ScienceDirect (considerando Journal of Systems and Software, Journal of System Architecture e Information and Software Technology).

Considerando os problemas relacionados ao processo de medição e/ou às medidas que impactam na implementação do controle estatístico de processos mencionados nas publicações, foram registrados 18 achados, listados na tabela 1:

Tabela 1 - Lista de achados do tipo Problemas

\begin{tabular}{|c|l|}
\hline Id & Problema \\
\hline P1 & Agrupamento de dados de projetos não similares. \\
\hline P2 & Base de medição mal estruturada. \\
\hline P3 & Coleta de uma mesma medida em momentos diferentes da execução dos processos nos projetos. \\
\hline P4 & Dados agregados. \\
\hline P5 & Dados ambíguos. \\
\hline P6 & Dados armazenados em diversas fontes não integradas. \\
\hline P7 & Dados de uma mesma medida coletados com granularidades diferentes. \\
\hline P8 & Dados perdidos. \\
\hline P9 & Definição operacional deficiente das medidas. \\
\hline P10 & Insuficiência ou ausência de dados coletados para as medidas definidas. \\
\hline P11 & Insuficiência ou ausência de informações de contexto das medidas. \\
\hline
\end{tabular}




\begin{tabular}{|l|l|}
\hline Id & Problema \\
\hline P12 & Insuficiência ou ausência de medidas associadas aos processos. \\
\hline P13 & $\begin{array}{l}\text { Medidas associadas a processos muito Iongos (mesmo com a granularidade correta, a frequência de coleta é } \\
\text { baixa). }\end{array}$ \\
\hline P14 & Medidas de alta granularidade. \\
\hline P15 & Medidas isoladas, sem que as medidas associadas, necessárias à análise, sejam coletadas. \\
\hline P16 & Medidas não alinhadas aos objetivos de negócio da organização. \\
\hline P17 & Medidas normalizadas incorretamente. \\
\hline P18 & Utilização de medidas de controle, ao invés de medidas de melhoria. \\
\hline
\end{tabular}

Analisando-se os dados extraídos das publicações selecionadas, percebeu-se que não há uma homogeneidade na identificação dos problemas relacionados ao processo de medição e/ou medidas que impactam na implementação do controle estatístico de processos.

Uma outra percepção obtida foi a diferença no nível de detalhamento das publicações ao citarem os problemas. Alguns autores generalizaram os problemas relacionados às medidas em um único problema: "medidas inúteis". Outros, por sua vez, não utilizam a expressão "medidas inúteis", mas, implicitamente, indicam, através dos problemas por eles relatados, a inutilidade das medidas. Por exemplo, o problema "medidas de alta granularidade", em uma análise geral, caracteriza "medidas inúteis".

Na pesquisa, 19,35\% das publicações relataram o problema "medidas inúteis", que, apesar de não se encontrar explícito na lista de achados de problemas, é implicitamente representado pelos demais problemas que caracterizam a inutilidade das medidas.

"Insuficiência ou ausência de informações de contexto das medidas" e "definição operacional deficiente das medidas" foram os problemas mais citados nas publicações, sendo seguidos por "medidas isoladas, sem que as medidas associadas, necessárias à análise, sejam coletadas", o que torna possível perceber que os problemas mais citados poderiam ser evitados em uma organização através de um programa de medição bem definido desde seus primeiros momentos.

"Dados perdidos" e "insuficiência ou ausência de dados coletados para as medidas definidas" também foram destacados, mostrando a necessidade de coleta e armazenamento constante dos dados para que as medidas possam ser utilizadas no controle estatístico de processos.

Apesar de alguns itens da lista de achados terem sido citados em um pequeno percentual das publicações, a importância dos mesmos não deve ser subestimada, como, por exemplo, o problema "utilização de medidas de controle, ao invés de medidas de melhoria", relevante para organizações que utilizam o controle estatístico de processos como apoio ao alcance de um nível mais elevado de maturidade em modelos como o MPS.BR (2007) e CMMI (2006).

Considerando as características relacionadas ao processo de medição e/ou às medidas que contribuem na implementação do controle estatístico de processos mencionadas nas publicações analisadas, foram registrados 21 achados, listados na tabela 2:

Tabela 2 - Lista de achados do tipo Características.

\begin{tabular}{|c|l|}
\hline Id & Característica \\
\hline C1 & Associação de medidas de processo com medidas de produto. \\
\hline C2 & Centralização dos dados coletados. \\
\hline C3 & Coleta automática das medidas. \\
\hline C4 & Coleta consistente das medidas. \\
\hline
\end{tabular}




\begin{tabular}{|l|l|}
\hline Id & Característica \\
\hline C5 & $\begin{array}{l}\text { Definição dos critérios que devem ser obedecidos para agrupar/comparar medidas coletadas nos projetos da } \\
\text { organizaço. }\end{array}$ \\
\hline C6 & Definição e coleta de medidas de produto e processo. \\
\hline C7 & Definição e coleta de medidas orientadas às tomadas de decisão. \\
\hline C8 & $\begin{array}{l}\text { Definição e coleta, desde o início das atividades de medição, de medidas relacionadas ao desempenho dos } \\
\text { processos. }\end{array}$ \\
\hline C9 & Existência de medidas de um processo de apoio quando o processo primário não possuir medidas suficientes. \\
\hline C10 & Existência de pelo menos 20 valores para cada medida a ser utilizada no controle estatístico de processos. \\
\hline C11 & Identificação dos relacionamentos entre as medidas. \\
\hline C12 & Medidas associadas a atividades que produzam itens tangíveis. \\
\hline C13 & Medidas associadas aos processos críticos. \\
\hline C14 & Medidas coletadas ao longo de todo o processo de desenvolvimento. \\
\hline C15 & Medidas coletadas para um fim específico, conhecido pelos envolvidos. \\
\hline C16 & Medidas de controle e melhoria de processo. \\
\hline C17 & Medidas passíveis de normalização, para possibilitar comparações. \\
\hline C18 & Medidas relacionadas às características de qualidade dos produtos. \\
\hline C19 & Registro preciso dos dados coletados para as medidas. \\
\hline C20 & Identificação de conjuntos de dados homogêneos. \\
\hline C21 & Utilização integrada de medidas de processo, projeto e produto. \\
\hline
\end{tabular}

A característica "coleta consistente das medidas" foi a mais citada pelas publicações, aparecendo em $45,16 \%$ delas. Esse alto índice pode ser explicado pela ampla conotação que a característica apresenta (várias (sub)características podem ser consideradas para que se obtenha uma coleta consistente das medidas) .

Também foi destacada a característica "definição dos critérios que devem ser obedecidos para agrupar/comparar medidas coletadas nos projetos da organização". Alguns autores destacaram a importância dessa característica ao afirmarem que, em algumas organizações, apesar de haver volume de dados e medidas suficientes, o agrupamento dos mesmos de forma não adequada fornece informações que baseiam decisões equivocadas. Em outros casos, organizações que não possuem o volume de dados necessários para aplicar as técnicas estatísticas reúnem diversas medidas inadequadamente, para obter o volume de dados necessário.

Outra característica reconhecida foi a "identificação dos relacionamentos entre as medidas" que, no controle estatístico de processos, apóia a interpretação, análise e investigação de situações demonstradas no comportamento dos processos.

Após terem sido obtidas a lista de problemas e a lista de características relacionadas ao processo de medição e/ou medidas que impactam na implementação do controle estatístico de processos, foi elaborada uma lista de requisitos necessários às medidas para utilização no controle estatístico de processos. Foram identificados os 20 requisitos listados na tabela 3 :

Tabela 3 - Lista de Requisitos identificados.

\begin{tabular}{|l|l|}
\hline Id & Requisito \\
\hline R1 & Alinhamento a objetivo(s) do projeto e da organização. \\
\hline R2 & Apoio à melhoria de processo. \\
\hline R3 & Associação a atividade(s) que produz(em) item(ns) tangível(is). \\
\hline R4 & Associação a processo crítico. \\
\hline R5 & Baixa granularidade. \\
\hline R6 & Consistência dos dados coletados. \\
\hline R7 & Critérios para agrupamento/comparação da medida definidos. \\
\hline R8 & Definição operacional correta e satisfatória. \\
\hline R9 & Existência das informações de contexto da medida. \\
\hline
\end{tabular}




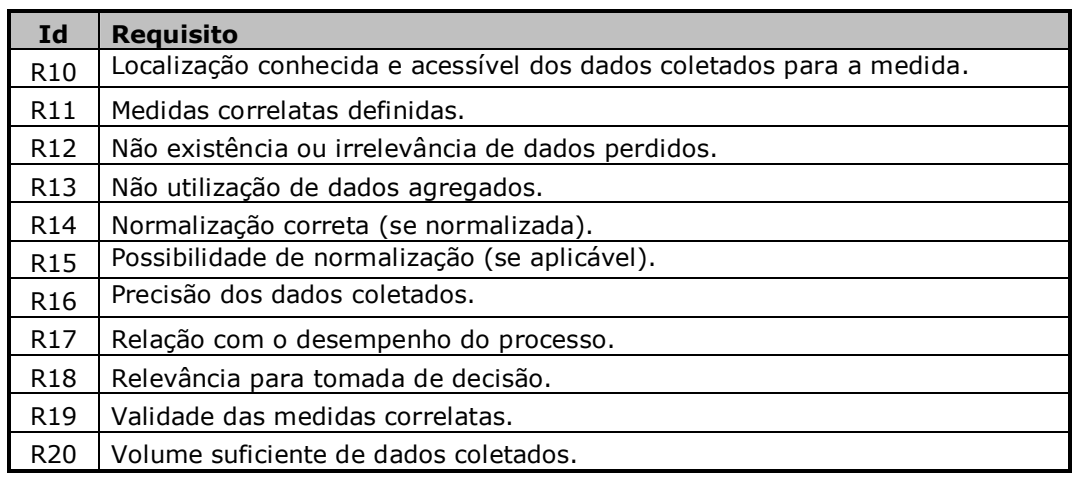

A tabulação detalhada dos dados da pesquisa, bem como a descrição completa do protocolo de pesquisa, seu teste e a lista de publicações analisadas podem ser encontradas em [Barcellos 2008].

Com base no conjunto de requisitos identificados foi definido um instrumento (checklist) para avaliação das bases de medidas. A figura 1 apresenta esse checklist.

\begin{tabular}{|c|c|c|c|c|}
\hline \multirow{2}{*}{$\begin{array}{l}\text { Requisitos } \\
\text { 1. A definição operacional da medida é correta e satisfatória. }\end{array}$} & \multicolumn{4}{|c|}{ Avaliação } \\
\hline & $($ ) atende & ( ) não atende & $\begin{array}{l}\text { ( ) atende } \\
\text { parcialmente }\end{array}$ & ( ) NFPA \\
\hline \multicolumn{5}{|l|}{ A definição operacional da medida inclui: } \\
\hline 1.1 Definição da medida & ( ) $\operatorname{sim}$ & ( ) não & & ( ) NFPA \\
\hline 1.2 Entidade medida & ( ) $\operatorname{sim}$ & ( ) não & & ( ) NFPA \\
\hline 1.3 Atributo medido & ( ) $\operatorname{sim}$ & ( ) não & & ( ) NFPA \\
\hline 1.4 Unidade de medida & ( ) $\operatorname{sim}$ & ( ) não & & ( ) NFPA \\
\hline 1.5 Tipo de dados (real, inteiro, etc) & $(\mathrm{)} \operatorname{sim}$ & ( ) não & & ( ) NFPA \\
\hline 1.6 Intervalo esperado dos dados & ( ) $\operatorname{sim}$ & ( ) não & & ( ) NFPA \\
\hline 1.7 Fórmula(s) & () $\operatorname{sim}$ & ( ) não & & $($ ) NFPA \\
\hline 1.8 Descrição precisa do procedimento de coleta & ( ) $\operatorname{sim}$ & ( ) não & & ( ) NFPA \\
\hline 1.9 Descrição precisa do procedimento de análise & ( ) $\operatorname{sim}$ & ( ) não & & ( ) NFPA \\
\hline $\begin{array}{l}\text { 2. A medida está alinhada a objetivo(s) do(s) projeto(s) e da } \\
\text { organização. }\end{array}$ & $(\mathrm{l})$ atende & ( ) não atende & $\begin{array}{l}\text { ( ) atende } \\
\text { parcialmente }\end{array}$ & $($ ) NFPA \\
\hline \multicolumn{5}{|l|}{ A medida está associada a: } \\
\hline 2.1 Objetivo do projeto & ( ) $\operatorname{sim}$ & ( ) não & & ( ) NFPA \\
\hline 2.2 Objetivo da organização & ( ) $\operatorname{sim}$ & ( ) não & & ( ) NFPA \\
\hline $\begin{array}{l}\text { 3. Os resultados da análise da medida são relevantes às } \\
\text { tomadas de decisão. }\end{array}$ & $($ ) atende & ( ) não atende & $\begin{array}{l}\text { ( ) atende } \\
\text { parcialmente }\end{array}$ & ( ) NFPA \\
\hline 4. A medida está relacionada ao desempenho de processo. & ( ) atende & ( ) não atende & $\begin{array}{l}\text { ( ) atende } \\
\text { parcialmente }\end{array}$ & ( ) NFPA \\
\hline 5. A medida está associada a um processo crítico. & $(\mathrm{)})$ atende & ( ) não atende & $\begin{array}{l}\text { ( ) atende } \\
\text { parcialmente }\end{array}$ & ( ) NFPA \\
\hline $\begin{array}{l}\text { 6. Os resultados da análise da medida são úteis à melhoria de } \\
\text { processo. }\end{array}$ & $(\mathrm{l})$ atende & ( ) não atende & $\begin{array}{l}\text { ( ) atende } \\
\text { parcialmente }\end{array}$ & ( ) NFPA \\
\hline $\begin{array}{l}\text { 7. A medida está associada a uma atividade que produz } \\
\text { item(ns) tangível(is). }\end{array}$ & $(\mathrm{l})$ atende & ( ) não atende & $\begin{array}{l}\text { ( ) atende } \\
\text { parcialmente }\end{array}$ & ( ) NFPA \\
\hline 8. As medidas correlatas à medida estão identificadas. & ( ) atende & ( ) não atende & $\begin{array}{l}\text { ( ) atende } \\
\text { parcialmente }\end{array}$ & ( ) NFPA \\
\hline 9. As medidas correlatas à medida são válidas. & $(\mathrm{l})$ atende & ( ) não atende & $\begin{array}{l}\text { ( ) atende } \\
\text { parcialmente }\end{array}$ & ( ) NFPA \\
\hline 10. A medida possui baixa granularidade. & ( ) atende & ( ) não atende & $\begin{array}{l}\text { ( ) atende } \\
\text { parcialmente }\end{array}$ & ( ) NFPA \\
\hline 11. A medida é passível de normalização (se aplicável). & $(\mathrm{l})$ atende & ( ) não atende & $\begin{array}{l}\text { ( ) atende } \\
\text { parcialmente }\end{array}$ & ( ) NFPA \\
\hline 12. A medida está normalizada corretamente (se aplicável). & ( ) atende & ( ) não atende & $\begin{array}{l}\text { ( ) atende } \\
\text { parcialmente }\end{array}$ & ( ) NFPA \\
\hline 13. A medida não considera dados agregados. & $(\mathrm{l})$ atende & ( ) não atende & $\begin{array}{l}\text { ( ) atende } \\
\text { parcialmente }\end{array}$ & ( ) NFPA \\
\hline $\begin{array}{l}\text { 14. Os critérios para agrupamento/comparação da medida } \\
\text { estão definidos. }\end{array}$ & $(\mathrm{l})$ atende & ( ) não atende & $\begin{array}{l}\text { ( ) atende } \\
\text { parcialmente }\end{array}$ & ( ) NFPA \\
\hline $\begin{array}{l}\text { 15. As informações de contexto da medida estão } \\
\text { armazenadas. }\end{array}$ & $(\mathrm{l})$ atende & ( ) não atende & $\begin{array}{l}\text { ( ) atende } \\
\text { parcialmente }\end{array}$ & ( ) NFPA \\
\hline \multicolumn{5}{|l|}{ É possível identificar: } \\
\hline 15.1 Momento da coleta & ) $\operatorname{sim}$ & ( ) não & & ( ) NFPA \\
\hline
\end{tabular}




\begin{tabular}{|c|c|c|c|c|}
\hline Requisitos & & & iação & \\
\hline $\begin{array}{l}15.2 \text { Condições da coleta (dados relevantes sobre a } \\
\text { execução do processo/projeto no momento da coleta da } \\
\text { medida) }\end{array}$ & () $\operatorname{sim}$ & ( ) não & & $($ ) NFPA \\
\hline 15.3 Executor da coleta & $($ ) $\operatorname{sim}$ & ( ) não & & () NFPA \\
\hline 15.4 Características do projeto no qual a medida foi coletada & ( ) $\operatorname{sim}$ & ( ) não & & () NFPA \\
\hline $\begin{array}{l}\text { 16. Os dados coletados para a medida têm localização } \\
\text { conhecida e acessível. }\end{array}$ & ( ) atende & ( ) não atende & $\begin{array}{l}\text { ( ) atende } \\
\text { parcialmente }\end{array}$ & $(\quad)$ NFPA \\
\hline 17. Há volume suficiente de dados coletados. & ( ) atende & ( ) não atende & $\begin{array}{l}\text { ( ) atende } \\
\text { parcialmente }\end{array}$ & ( ) NFPA \\
\hline $\begin{array}{l}\text { 18. Não há dados perdidos para a medida ou a quantidade de } \\
\text { dados perdidos é irrelevante. }\end{array}$ & ( ) atende & ( ) não atende & $\begin{array}{l}\text { ( ) atende } \\
\text { parcialmente }\end{array}$ & ( ) NFPA \\
\hline 19. Os dados coletados são precisos. & ( ) atende & ( ) não atende & $\begin{array}{l}\text { ( ) atende } \\
\text { parcialmente }\end{array}$ & ( ) NFPA \\
\hline 20. Os dados coletados são consistentes. & $(\mathrm{l})$ atende & ( ) não atende & $\begin{array}{l}\text { ( ) atende } \\
\text { parcialmente }\end{array}$ & ( ) NFPA \\
\hline Características dos dados coletados: & & & & \\
\hline $\begin{array}{l}20.1 \text { Os dados foram coletados no mesmo momento da } \\
\text { execução do processo ao longo dos projetos. }\end{array}$ & ( ) $\operatorname{sim}$ & ( ) não & & $(\quad)$ NFPA \\
\hline 20.2 Os dados foram coletados sob as mesmas condições. & ( ) $\operatorname{sim}$ & ( ) não & & $(\quad)$ NFPA \\
\hline 20.3 Os dados compõem grupos relativamente homogêneos. & () $\operatorname{sim}$ & ( ) não & & () NFPA \\
\hline
\end{tabular}

NFPA $=$ Não foi possível avaliar

Figura 1 - Checklist para avaliação de bases de medidas.

\section{Experiência de Avaliação de Bases de Medidas}

Após a elaboração do checklist, este foi utilizado em duas empresas do Rio de Janeiro, como uma primeira avaliação de sua aplicabilidade e utilidade.

Inicialmente, o checklist foi aplicado individualmente a cada medida selecionada na base de medidas das organizações. Em seguida, as medidas foram agrupadas para uma análise por processo.

Avaliação da Base de Medidas da Organização "A”

(i) Contexto: Os dados presentes na base de medidas da organização $A$ foram coletados ao longo de projetos realizados nessa organização, avaliada CMMI nível 2 e que encontra-se implementando as práticas necessárias ao atendimento dos requisitos do nível 3 do modelo. Antes da avaliação da base de medidas, foram realizadas reuniões que permitiram que os principais processos, práticas e ferramentas da organização fossem conhecidos.

(ii) Análise Geral: A organização A possui um plano de medição detalhado, que, recentemente, foi adequado para atender os requisitos do nível 3 do CMMI. As medidas definidas no plano encontram-se alinhadas ao planejamento estratégico organizacional. Os projetos são agrupados de acordo com suas características, permitindo analisar os valores coletados para as medidas entre projetos de um mesmo grupo. A empresa possui um ambiente de apoio à gerência dos projetos que permite que algumas das medidas definidas sejam coletadas automaticamente ou através do próprio ambiente. Outras são registradas manualmente em planilhas.

Apesar da organização não possuir um banco de dados com uma estrutura refinada para a base de medidas e das medidas coletadas serem armazenadas em locais distintos (planilhas e banco de dados), a organização realiza a integração dos dados coletados centralizando-os em planilhas de projetos e organizacional, vinculadas entre si.

Considerando que a coleta das medidas despende esforço e, consequentemente, tem um custo para a organização, ao definir o plano de medição, a organização $A$ 
incluiu apenas as medidas que considerou indispensáveis. No momento em que o objetivo principal era alcançar o nível 2 do CMMI, foi definido um pequeno grupo de medidas capaz de atender aos requisitos do modelo e, ao mesmo tempo, ser útil à organização, tendo seus resultados aplicáveis em decisões e perceptíveis a todos os envolvidos, o que, naturalmente, motivaria a medição. À medida em que novas necessidades surgiram, novas medidas foram definidas. Quando o objetivo principal passou a ser alcançar o nível 3 do modelo, uma revisão refinada do plano de medição foi realizada para identificar as medidas necessárias às novas práticas da organização.

(iii) Análise Detalhada: Para realizar a avaliação foram utilizados dados de medidas coletadas pela organização em 2007, tendo sido consideradas apenas as medidas referentes a processos diretamente relacionados ao desenvolvimento de software.

A organização A possui em sua base de medidas um volume considerável de dados que foram coletados em mais de quarenta projetos desde 2003, porém, muitos desses dados não podem ser utilizados no controle estatístico de processos, pois referem-se a definiçõos diferentes de um mesmo processo. Por esse motivo, decidiu-se considerar apenas os dados de projetos realizados em 2007, onde foram utilizadas as mesmas definições para os processos dos projetos.

Considerando as medidas referentes a processos relacionados ao desenvolvimento de software coletadas em 2007, neste estudo foram avaliadas 61 medidas. Na base de medidas dessa empresa foram encontradas medidas como: esforço planejado, esforço realizado, esforço consumido, $\mathrm{n}^{\mathrm{o}}$ de itens planejados, $\mathrm{n}^{\mathrm{o}}$ de itens realizados, itens com desvio de prazo, $\mathrm{n}^{\circ}$ de requisitos incluídos, $\mathrm{n}^{\circ}$ de requisitos excluídos, $\mathrm{n}^{\mathrm{o}}$ de requisitos alterados, $\mathrm{n}^{\circ}$ de não conformidades, $\mathrm{n}^{\circ}$ de defeitos identificados internamente, $\mathrm{n}^{\mathbf{0}}$ de defeitos identificados pelo cliente, $\mathrm{n}^{\mathrm{o}}$ total de não conformidades registradas, $\mathrm{n}^{\circ}$ de não conformidades concluídas e esforço despendido em correção de não conformidades, que, conforme sua definição operacional no plano de medição, seriam coletadas/analisadas ao final de cada fase ou mês, o que caracteriza uma granularidade muito alta para aplicação no controle estatístico de processos.

Porém, percebeu-se que a organização, devido ao apoio provido pelo ambiente de gerência de projetos utilizado, manteve o registro de algumas dessas medidas em granularidade menor. Por exemplo, algumas medidas relacionadas a prazo e esforço, apesar de, para atender os requisitos dos níveis iniciais do CMMI poderem ser coletadas/analisadas mensalmente, os registros por dia, semana e atividade foram mantidos, podendo ser recuperados.

Além disso, mesmo que, no plano de medição, as medidas não estivessem explicitamente relacionadas a processos específicos, os dados foram coletados $\mathrm{e}$ armazenados de tal forma que foi possível identificar o valor das medidas para cada processo.

Durante a aplicação do checklist a cada medida, notou-se que o requisito referente à identificação das medidas correlatas não foi atendido por nenhuma medida. Além disso, outros requisitos importantes como existência das informações de contexto e definição dos critérios de agrupamento/comparação das medidas também não foram atendidos.

Além disso, não há volume de dados suficiente para o controle estatístico para a maioria das medidas (ressaltando que, conforme já mencionado, foram considerados apenas os dados de projetos realizados em 2007). 
A análise dos dados da base de medidas da organização $A$ confirmou a afirmação de alguns autores, como Boria (2007) e Kitchenham et al. (2007), de que as medidas utilizadas para atender os requisitos dos níveis iniciais do CMMI não são aplicáveis ao controle estatístico de processos. Como diagnóstico geral da avaliação, concluiu-se que as medidas da base de medidas da organização $A$ precisam de adequações para que possam ser utilizadas no controle estatístico de processos. Algumas das medidas coletadas são inúteis ao controle estatístico de processos como, por exemplo, número de projetos aderentes ao plano de medição. Outras, considerando os dados coletados, têm aplicabilidade, mas, sozinhas, não são suficientes para a realização do controle estatístico de processos pois, mesmo que seus dados sejam utilizados em técnicas estatísticas, a análise será muito difícil ou, até, impraticável, uma vez que informações de contexto e medidas correlatas não estão disponíveis. Além disso, a utilização apenas das medidas existentes não é capaz de descrever o desempenho dos processos.

Baseando-se nos resultados da avaliação, algumas ações que devem ser realizadas pela organização para adequar sua base de medidas aos requisitos necessários para realização do controle estatístico de processos são: refinar o plano de medição a fim de relacionar, formalmente, as medidas aos processos; organizar a base de medidas conforme alterações no plano de medição; definir e coletar medidas correlatas às medidas definidas; registrar informações de contexto das medidas, incluindo informações relevantes sobre a execução do processo e projeto no momento da coleta da medida; refinar a caracterização dos projetos e definir critérios para agrupamento/comparação de medidas; definir e coletar outras medidas relacionadas ao desempenho e melhoria dos processos ${ }^{3}$; coletar volume de dados suficiente para as medidas.

\section{Avaliação da Base de Medidas da Organização " $B$ ”}

(i) Contexto: Os dados presentes na base de medidas da organização $B$ foram coletados ao longo de projetos realizados na organização, também avaliada CMMI e usuária do Ambiente TABA [Villela 2004]. A avaliação da base de medidas foi realizada sem interação com os membros da organização, ou seja, para a realização da avaliação foram consideradas, exclusivamente, as informações contidas na base de medidas e o conhecimento pessoal relativo aos ambientes, processos e estrutura do Ambiente TABA.

(ii) Análise Geral: A organização $B$ possui um plano de medição bastante refinado cujas medidas definidas encontram-se alinhadas aos objetivos de software estabelecidos pela organização. No entanto, não foi possível avaliar o planejamento estratégico da organização para analisar o alinhamento dos objetivos de software estabelecidos aos objetivos definidos no planejamento estratégico, uma vez que tais informações não se encontravam na base de medidas.

Os projetos da organização são caracterizados seguindo o mecanismo de caracterização presente no Ambiente TABA, que inclui os critérios: indústria, tipo de software, paradigma, natureza do projeto, nível de experiência do gerente, nível de experiência da equipe, nível de experiência dos clientes, uso de tecnologia inovadora,

\footnotetext{
3 Apesar das medidas analisadas terem relação com o desempenho dos processos, a maioria delas está mais relacionada a controle que melhoria.
} 
restrição de cronograma, restrição de desempenho, restrição de segurança, restrição de recursos humanos e distribuição geográfica da equipe.

Na organização $B$, o plano de medição é elaborado através da identificação dos objetivos de software e, a partir deles, da seleção de questões e medidas a eles associadas. Essas medidas são relacionadas a processos que compõem o processo padrão da organização. Em cada projeto, o plano de medição é instanciado e novas medidas, necessárias ao atendimento de objetivos específicos do projeto, podem ser adicionadas.

(iii) Análise Detalhada: Para realizar a avaliação foram utilizados dados de medidas coletadas pela organização em 2005, considerando 7 projetos. Foram avaliadas 124 medidas, associadas aos processos: gerência de requisitos, gerência de riscos, planejamento do projeto, gerência de configuração, medição e análise, garantia da qualidade, definição de requisitos, solução técnica, verificação, validação, análise de decisão e resolução, integração do produto, definição do processo organizacional, foco no processo organizacional e gerência integrada do projeto. Algumas das medidas avaliadas foram: esforço planejado, esforço realizado, tempo estimado, tempo real, $\mathrm{n}^{\mathbf{o}} \mathrm{de}$ não conformidades, $\mathrm{n}^{\circ}$ de requisitos incorretos, $\mathrm{n}^{\circ}$ de requisitos ambíguos, $\mathrm{n}^{\circ}$ de riscos identificados, $\mathrm{n}^{\mathrm{o}}$ de riscos identificados que ocorreram e $\mathrm{n}^{\mathrm{o}}$ de erros, entre outras.

Apesar da organização possuir medidas com definições operacionais satisfatórias, alinhadas aos objetivos e relacionadas a todos os processos executados, o maior problema percebido foi a alta granularidade das medidas. A maior parte das medidas foi coletada ao final das fases ou por macroatividade nos projetos, uma vez que sua definição e coleta foram baseadas, principalmente, na necessidade de atender aos requisitos dos níveis iniciais do CMMI. A baixa frequiência da coleta também levou ao não atendimento do requisito volume de dados suficiente. Considerando que foram analisados dados de 7 projetos, algumas medidas, que foram coletadas uma única vez em cada projeto, não apresentaram volume de dados suficiente para aplicação do controle estatístico de processos. Além disso, faltam informações de contexto sobre as medidas. $\mathrm{O}$ registro do valor coletado para as medidas contém informações sobre o momento da coleta, porém, essas informações não são suficientes para a aplicação do controle estatístico de processos.

Alguns pontos positivos percebidos durante a avaliação foram: presença de medidas correlatas, raras situações de dados não coletados para as medidas, grupos de dados relativamente homogêneos, presença de medidas para todos os processos e relevância das medidas às tomadas de decisão.

Assim como ocorreu na análise dos dados da base de medidas da organização $A$, a análise da base de medidas da organização $B$ gerou resultados que vão ao encontro da afirmação de que as medidas utilizadas para atender os requisitos dos níveis iniciais do CMMI não são aplicáveis ao controle estatístico de processos. A base de medidas $B$ mostra-se aderente aos requisitos dos níveis iniciais do CMMI, porém, suas medidas ainda não são aplicáveis para o controle estatístico de processos.

Como diagnóstico geral, conclui-se que a base de medidas $B$ precisa de algumas adequações para que suas medidas sejam úteis ao controle estatístico de processos. A principal delas relaciona-se à frequiência de coleta das medidas, o que impacta diretamente em sua granularidade. Há medidas associadas a todos os processos e há um número satisfatório de medidas definidas, o que contribui para a seleção dos processos 
críticos a serem submetidos ao controle estatístico de processos, porém, para isso, novos dados devem ser coletados, com a granularidade adequada, a fim de produzir o volume de dados necessário às medidas.

Baseando-se nos resultados da avaliação, algumas ações que devem ser realizadas pela organização $B$ para adequar sua base de medidas aos requisitos necessários para realização do controle estatístico de processos são: revisar a definição das medidas considerando menor granularidade; incluir nas informações de contexto das medidas coletadas informações relevantes sobre a execução do processo e projeto no momento da coleta da medida; e coletar volume de dados suficiente para as medidas.

O Ambiente TABA, ao apoiar a organização $B$ na definição e execução do plano de medição, contribuiu para um cenário mais propício à aplicação do controle estatístico de processos do que o cenário diagnosticado na organização $A$. Em comparação à base de medidas $A$, a base de medidas $B$ precisará de menos adequações para atender aos requisitos para aplicação do controle estatístico de processos.

\section{Conclusão}

O controle estatístico de processos contém diversas ferramentas de apoio à análise do comportamento de processos que são utilizadas para alcançar a estabilidade dos processos e buscar sua melhoria contínua através da redução dos limites de variação. Sua utilização no domínio da manufatura é ampla, porém, a aplicação a processos de software ainda é recente.

O crescente interesse das organizações em elevar o grau de maturidade de seus processos e alcançar os níveis mais elevados de modelos de maturidade como MPS.BR (2007) e CMMI (2006) tem levado as organizações de software à utilização das técnicas do controle estatístico em seus processos.

Apesar do crescente número de estudos publicados no contexto da aplicação das técnicas do controle estatístico de processos a processos de software, há muito poucos registros que forneçam orientações práticas satisfatórias para a implementação do controle estatístico de processos, pois uma parte considerável dos estudos registrados foca evidenciar a possibilidade e vantagens da aplicação do controle estatístico de processos a processos de software ou propor abordagens utilizadas em processos de software baseadas nos princípios do controle estatístico. Uma vez que ainda não há um conjunto formal, consolidado e detalhado, de diretrizes para a realização do controle estatístico de processos em processos de software, as organizações que realizam sua implementação têm encontrado dificuldades.

Dentre as dificuldades que as organizações de software enfrentam para realizar o controle estatístico de processos, a não adequação de suas bases de medidas à aplicação das técnicas estatísticas tem sido destacada [Kitchenham et al. 2006] [Boria 2007]. Resolver essa questão é fundamental, pois, sem os dados adequados, o controle estatístico de processos não pode ser realizado de forma satisfatória. A identificação, seleção, coleta e armazenamento de medidas adequadas têm papel fundamental para iniciar a implementação do controle estatístico de processos para processos de software [Tarhan and Demirors, 2006].

Este artigo apresentou um instrumento para avaliação de bases de medidas considerando sua aplicabilidade para o controle estatístico de processos.

Algumas considerações sobre a experiência de avaliação de bases de medidas, relatada na seção 4, são pertinentes e necessárias. Para realizar a avaliação, antes de 
aplicar o checklist, foi necessária uma análise global do plano de medição e da base de medidas das organizações. Também foi necessário avaliar as medidas no contexto dos processos aos quais estavam relacionadas. A avaliação das medidas individualmente através do checklist não se mostrou suficiente para determinar a aplicabilidade ou não para o controle estatístico de processos. Sendo assim, conclui-se que o instrumento para avaliação de medidas proposto deve considerar, além da avaliação individual das medidas, a avaliação no contexto dos processos e da base de medidas como um todo.

O trabalho descrito neste artigo está relacionado a uma tese de doutorado em andamento. Sendo assim, uma evolução do instrumento proposto contemplando os aspectos ressaltados durante a experiência de avaliação das bases de medidas será realizada. Essa evolução deve incluir a avaliação das medidas no contexto dos processos e da base de medidas como um todo, bem como a definição do procedimento a ser utilizado para concluir, a partir dos resultados da avaliação, se uma medida é aplicável ou não ao controle estatístico de processos. O procedimento deve considerar a criticidade dos requisitos à realização do controle estatístico de processos e, com base nos requisitos atendidos e não atendidos por uma medida, indicar seu grau de aplicabilidade. Para identificar a criticidade de cada requisito à aplicação do controle estatístico de processos, pode-se considerar os registros da literatura (sabe-se, por exemplo, que ter baixa granularidade é condição fundamental para que uma medida possa ser aplicada ao controle estatístico de processos) e realizar testes incluindo a aplicação das técnicas estatísticas em dados coletados para medidas que não atendam a um requisito específico, a fim de analisar as consequências do não atendimento de um determinado requisito. Além disso, a evolução do instrumento incluirá uma definição detalhada de cada requisito, bem como orientações sobre como cada um deles deve ser avaliado.

\section{Referências Bibliográficas}

Barcellos, M. P., 2008, "Uma Abordagem para Controle Estatístico de Processos de Software em Organizações de Alta Maturidade”, Exame de Qualificação para Tese de D.Sc., COPPE/UFRJ, Rio de Janeiro, Brasil.

Biolchini, J., Mian, P. G., Natali, A. C., 2005, "Systematic Review in Software Engineering", RT-ES 679/05, COPPE/UFRJ, Rio de Janeiro, Brasil.

Boria, J. L., 2007, “What’s Wrong With My Level 4?”, Comunicação Pessoal.

Card, D. N.: Statistical Techniques for Software Engineering Practice. Proceedings of the 26th International Conference on Software Engineering, pp. $722-723$ (2004).

CMMI - Capability Maturity Model Integration, 2006, SEI - Software Engineering Institute, CMU - Carnegie Mellon University, v 1.2.

Eickelmann, N., Anant, A., 2003, "Statistical Process Control: What You Don't Measure Can Hurt You", IEEE Software, Volume 20, Issue 2, pp. 40 - 51.

Fenton, N., Marsh W., Neil, M., Cates, P., Forey, S., Tailor, M.:, 2004, "Making Resource Decisions for Software Projects", Proceedings of the 26th International Conference on Software Engineering - ICSE'04, pp. 397-406.

Fuggetta, A., 2000, "Software Process: A Roadmap", In: Proceedings of the $22^{\text {nd }}$ International Conference on Software Engineering, Limerick, Irlanda, pp. 25-34.

Garcia, F., Serrano, M., Cruz-Lemos, J., Ruiz, F., Piattini, M., 2007, "Managing Software Process Measurement: A Metamodel-Based Approach"Information Sciences, Volume 177, no 12, pp. 2570-2586. 
ISO/IEC, ISO/IEC TR 15504-2003, 2003, Information Technology - Software Process Assessment, International Organization for Standardization and the International Electrotechnical Commission, Geneva, Switzerland.

ISO/IEC FDIS 15939 - 2002 (E) Software Engineering - Software Measurement Process, International Organization for Standardization and the International Electrotechnical Commission, Geneva, Switzerland.

Kilpi, T., 2001, "Implementing a Software Metrics Program at Nokia", IEEE Software, Volume 18, Issue 6, pp. 72-77.

Kitchenhan, B., Kutay, C., Jeffery, R., Connaughton, C., 2006, "Lessons Learnet from the Analysis of Large-scale Corporate Databases", Proceedings of the 28th International Conference on Software Engineering - ICSE'06, pp. 439-444.

Kitchenhan, B., Kutay, C., Jeffery, R., Connaughton, C., 2007, "Misleading Metrics and Unsound Analyses", IEEE Software, Volume 24, Issue 2, pp. 73 - 78.

Komuro, M., 2006, "Experiences of Applying SPC Techniques to Software Development", Proceedings of the 28th International Conference on Software Engineering - ICSE'06, pp. 577-584.

Lantzy, M. A., 1992, "Application of Statistical Process Control to the Software Process", Proceedings of the 9th Washington Ada Symposium on Empowering Software Users and Developers, ACM Press, pp. 113-123.

Montoni, M., 2007, "Uma Abordagem para Condução de Iniciativas de Melhoria de Processos de Software", Exame de Qualificação para o Doutorado, COPPE/UFRJ, Rio de Janeiro, Brasil.

MPS.BR - Melhoria de Processo do Software Brasileiro, Guia Geral, v. 1.2, 2007.

Sargut, K. U., Demirors, O., 2006, "Utilization of Statistical Process Control (SPC) in Emergent Software Organizations: Pitfalls and Suggestions", Software Quality Journal, pp. 135-157.

Tarhan, A., Demirors, O., 2006, "Investigating Suitability of Software Process and Metrics for Statistical Process Control", Lectures Notes in Computer Science, Volume 4257/2006, pp. 88-99.

Villela, K., 2004, "Definição e Construção de Ambientes de Desenvolvimento de Software Orientados à Organização”, Tese de D. Sc., COPPE/UFRJ, Rio de Janeiro, Brasil.

Wang, Q., Li, M., 2005, "Measurement and Improving Software Process in China”, Proceedings of International Symposium on Empirical Software Engineering ISESE 2005, pp.183-192.

Wang, Q., Jiang, N, Gou, L., Liu, X., Li, M., Wang, Y., 2006, "BSR: A StatisticalBased Approach for Establishing and Refining Software Process Performance Baseline”, International Conference on Software Engineering - ICSE'06, pp. 585594.

Wheeler, D. J., Poling, R. S., 1998, "Building Continual Improvement: A Guide for Business", SPC Press. 